\title{
Magnetic Domain Structure of MnAs Thin Films as a Function of Temperature
}

\author{
Masaki Mizuguchi ${ }^{1,2}$, Hiromi Kuramochi ${ }^{2,3}$, Jun Okabayashi ${ }^{2,4}$, Takashi Manago ${ }^{1,2}$, \\ and Hiroyuki Akinaga ${ }^{1,2}$ \\ ${ }^{1}$ Research Consortium for Synthetic Nano-Function Materials Project-National Institute of Advanced Industrial Science and Technology, \\ Tsukuba 305-8568, Japan \\ ${ }^{2}$ Nanotechnology Research Institute-National Institute of Advanced Industrial Science and Technology, Tsukuba 305-8562, Japan \\ ${ }^{3}$ Research Consortium for Synthetic Nano-Function Materials Project-Seiko Instruments, Tsukuba, 305-8562, Japan \\ ${ }^{4}$ Research Consortium for Synthetic Nano-Function Materials Project-The University of Tokyo, Tokyo 113-8656, Japan
}

We have investigated magnetic domain structures of MnAs thin films grown on GaAs substrates by a magnetic force microscope. We observed, by an atomic force microscope, rectangular defects along GaAs [110] direction which disperse randomly on the surface of MnAs/ GaAs (001). The Curie temperature of MnAs is $45^{\circ} \mathrm{C}$, and it is successfully confirmed directly by the variable temperature magnetic force microscope observation. We also investigated magnetic domain structures of MnAs/GaAs (111)B, and no apparent relation was observed between the topographic structure and the magnetic domain structure.

(Received July 16, 2003; Accepted September 25, 2003)

Keywords: MnAs, magnetic force microscopy, phase transition

\section{Introduction}

The epitaxial growth of magnetic thin films on semiconductor substrates such as a MnSb thin film on a GaAs substrate has received much attention since the manganese compounds have various magnetic properties. ${ }^{1-3)}$ A MnAs thin film can be epitaxially grown on a GaAs substrate by Molecular-Beam Epitaxy (MBE) as a ferromagnetic-metal/ semiconductor hybrid film. ${ }^{4-7)}$ Tanaka et al. have found that the starting monolayer template plays a critical role in determining the epitaxial relationship between MnAs thin film and the GaAs (001) substrate. ${ }^{5)}$ In bulk MnAs, the first order phase transition from the ferromagnetic phase with the NiAs-type hexagonal structure ( $\alpha$-MnAs) to the paramagnetic phase with the MnP-type orthorhombic structure $(\beta$ MnAs) is observed at around $45^{\circ} \mathrm{C}^{8)}$ It takes notice whether the phase transition can be identified at around the temperature or not even for the thin MnAs film.

Ferromagnetic materials generally form magnetic domain structures to reduce their magnetostatic energy. Magnetic force microscope (MFM) is a powerful tool to identify the shape of magnetic domain walls and the magnetic vortex directly. ${ }^{9)}$ MFM detects the magnetic force exerted on a magnetic probe tip when the tip is scanned over the surface of the sample, and magnetic change should be observed during the phase transition. In this paper, by using MFM, we investigated the temperature dependence of magnetic domain structures of MnAs films grown on GaAs substrates.

\section{Experimental}

MnAs layers were grown on semi insulating GaAs (001) substrates by MBE. After growing a GaAs buffer layer with the thickness of $10 \mathrm{~nm}$ at $580^{\circ} \mathrm{C}$, the substrate temperature was cooled down to $200^{\circ} \mathrm{C}$ to grow the MnAs film. The GaAs surface reconstruction changed from $2 \times 4$ to $\mathrm{c}(4 \times 4)$ for GaAs (001) and from $\sqrt{ } 19 \times \sqrt{ } 19$ to $2 \times 2$ for GaAs (111)B, which was checked by reflection high-energy electron diffraction (RHEED) patterns. Here, GaAs (111)B means the surface of GaAs terminated by the As layer. During the growth of MnAs, the flux ratio of $\mathrm{As}_{4} / \mathrm{Mn}$ was set at about 100. The thickness of MnAs film is designed to be $30 \mathrm{~nm}$. After the growth of MnAs, sample was annealed at $350^{\circ} \mathrm{C}$ under the As flux. From the X-ray diffraction and magnetization measurement, it is shown that the MnAs film is grown epitaxially with the growth plane of MnAs (1-100). ${ }^{5)}$ The $a$ axis of the hexagonal $\mathrm{NiAs}$ crystal structure, namely the magnetization easy axis of the thin film, is parallel to the [110] GaAs direction in the (001) plane. On the other hand, the growth plane of MnAs grown on GaAs (111)B is MnAs (0001), and a flat surface can be obtained.

Atomic Force Microscope (AFM) and MFM measurements were performed with an environmental control AFM/ MFM unit (SII, SPA-300HV) in vacuum $\left(<5 \times 10^{-2} \mathrm{~Pa}\right)$. It based on the optical lever system to measure the deflection of the cantilever. ${ }^{10)}$ The cantilever with a microfabricated Si tip is coated with $\mathrm{Al} / \mathrm{Co}$ alloy. The nominal spring constant is $42 \mathrm{~N} / \mathrm{m}$. Noncontact mode is used for the MFM observation, the tip is rastered at the height of $0-30 \mathrm{~nm}$ over the sample surface. Operation in vacuum without air resistance gains a larger $\mathrm{Q}$ factor and makes a high-sensitive observation possible. ${ }^{11)}$ MFM observations were carried out in the zero field after the magnetization of samples. In-plane magnetization of the sample was done using an electromagnet up to the magnetic field of $1.5 \mathrm{~T}$, which is enough for saturation, at room temperature. Sample temperature was varied from 25 to $55^{\circ} \mathrm{C}$ with a rate of $1^{\circ} \mathrm{C} / \mathrm{min}$ under $1 \times 10^{-1} \mathrm{~Pa}$. To observe the same area during phase transition, AFM and MFM images were taken simultaneously.

Magnetic properties of MnAs films grown on GaAs (001) was investigated by superconducting quantum interference device (SQUID). Polar magnetic circular dichroism (MCD) measurements in a visible wavelength region were also performed at room temperature. 
(a)

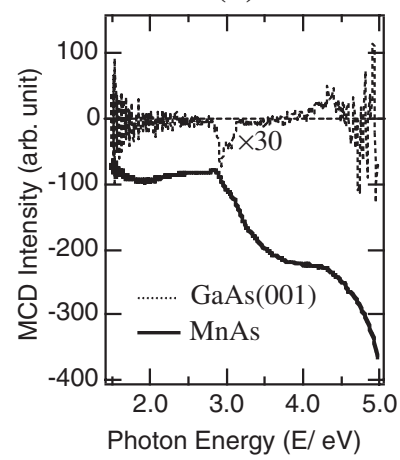

(b)

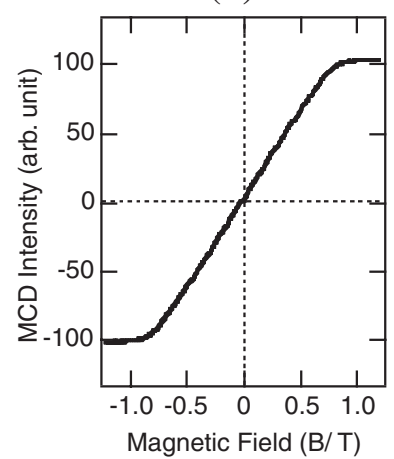

Fig. 1 (a) MCD spectrum of MnAs thin film with the thickness of $30 \mathrm{~nm}$ grown on GaAs (001). Enlarged spectrum of GaAs (001) is also shown as a reference. (b) MCD hysteresis of MnAs curve measured at room temperature. The wavelength of the incident light was set at $418 \mathrm{~nm}$.

\section{Results and Discussion}

\subsection{MnAs on GaAs (001)}

Magnetization measurements were performed to investigate the magnetic properties of the MnAs/GaAs (001) thin film by SQUID. The magnetic field was applied in-plane along different directions during measurements at room temperature. Square hysteresis loop was observed by applying the magnetic field in-plane along GaAs [110]. Completely different hysteresis characteristics were observed when the magnetic field was applied along GaAs [1-10]. This clearly shows the uniaxial anisotropy of the present sample and the easy axis of magnetization is in-plane, parallel to GaAs [110].

The magnetic properties of MnAs film grown on GaAs (001) were investigated by polar MCD experiments. Figure 1(a) shows the MCD spectrum of MnAs thin film taken at room temperature. A spectrum of GaAs (001) is also shown as a reference. Very large MCD is observed for MnAs, which indicates the thin film has a ferromagnetic behavior at room temperature. An MCD hysteresis curve measured at room temperature is shown in Fig. 1(b). It is found that the coercivity is very small for this film. This fact is explained by assumption that the easy magnetization axis of MnAs film grown on GaAs (001) lies in the in-plane of GaAs, and the hysteresis curve taken by the "polar" configuration shows very "soft" magnetization characteristics. This indicates that the MnAs film has a high crystallographic quality, and MnAs (1-100) was only grown selectively between the two types of plane, MnAs (1-100) and MnAs (1-101). ${ }^{5)}$

Figures 2(a) and (b) show topographic images of MnAs/ GaAs $(001)$ surface $\left(5 \times 5 \mu \mathrm{m}^{2}\right)$. Characteristic rectangular defects along GaAs [1-10] direction were observed randomly on the surface. They are $300-800 \mathrm{~nm}$ in length and $15-40 \mathrm{~nm}$ in depth. Roughness of this surface is about $2 \mathrm{~nm}$ and white dots ( $\sim 4 \mathrm{~nm}$ in height) covered the surface. Figures 2 (c) and (d) are MFM images corresponding to Figs. 2(a) and (b), respectively. They show remnant magnetic state after magnetization along different orientations. Produced stripe magnetic domains along GaAs [110] direction were observed after magnetization parallel to [110] direction, easy magnetization axis, as shown in Fig. 2(c). The domain structure was

(a)

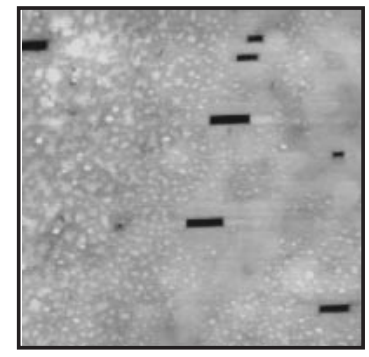

(c)

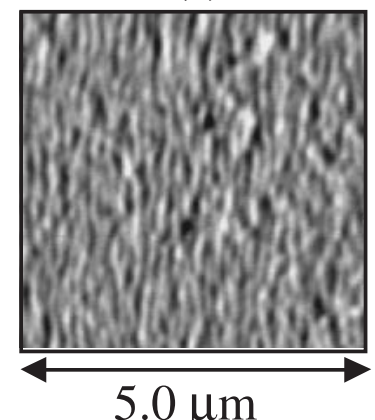

$5.0 \mu \mathrm{m}$ (b)

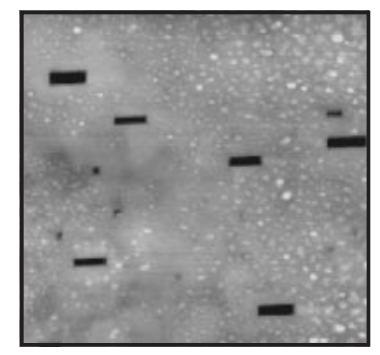

(d)

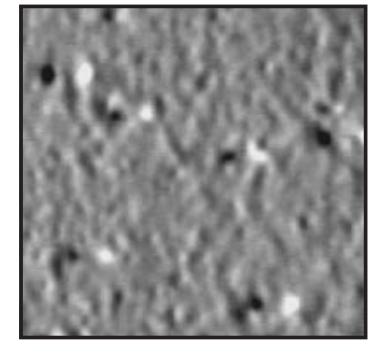

GaAs [1-10]

Fig. 2 Topographic images and Magnetic domain structure of MnAs/ GaAs (001) thin film. (a) and (b) Topographic images of $5 \times 5 \mu \mathrm{m}^{2}$ area (c) MFM image of the same area as (a) after magnetization along GaAs [110]. (d) MFM image corresponding to (b) after magnetization along GaAs [1-10]. All images were taken at $20^{\circ} \mathrm{C}$.

not affected by the magnetic tip, because it did not change according to scan direction. When the sample was magnetized parallel to GaAs [1-10], rotating the sample 90 degree in the electromagnet, stripe domains were not clearly observed. Pairs of black and white spots were also observed in Fig. 2(d). They are observed inverted using an MFM tip with opposite magnetization orientation. So that they are magnetic poles with outward (white) and inward-plane (black) magnetic moments. Comparing the MFM image [Fig. 2(d)] to the topographic image [Fig. 2(b)], it is found that these poles are located at the edges of rectangular defects. On the other hand, it is rather difficult to find pair of poles in Fig. 2(c). It is consistent with the M-H characteristics, conspicuous domain walls due to the larger remnant obscure the poles in the case of magnetization parallel to the easy magnetization axis. The difference between two MFM images obviously shows the in-plane anisotropy of the MnAs thin film in conformity to the $\mathrm{M}-\mathrm{H}$ characteristics. It is an example of magnetic structural change controlled by applying magnetic field.

The domain structure is governed by topography as with the previous work, ${ }^{12)}$ and it depends on the structural properties of the thin film, i.e., the surface orientation, thickness and defects. In the case of the present sample, the characteristic fair rectangular defects make local nonmagnetic areas on the surface.

Next, the change of magnetic domain structure was observed during the phase transition. After magnetization at room temperature, the sample temperature was increased up 
(a) AFM

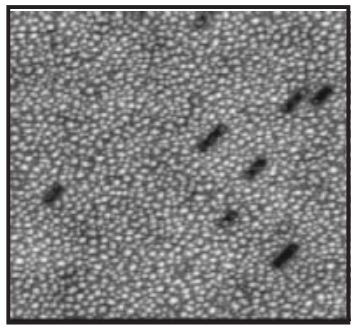

(c) $40^{\circ} \mathrm{C}$

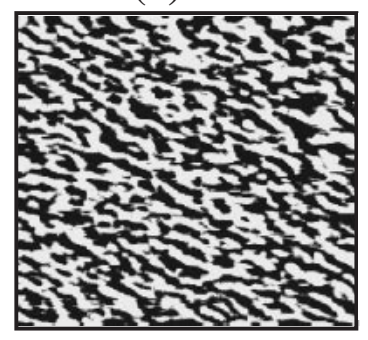

(e) $45^{\circ} \mathrm{C}$

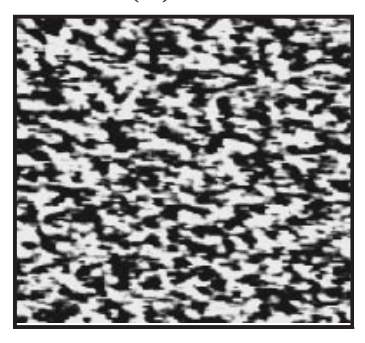

(g) $50^{\circ} \mathrm{C}$

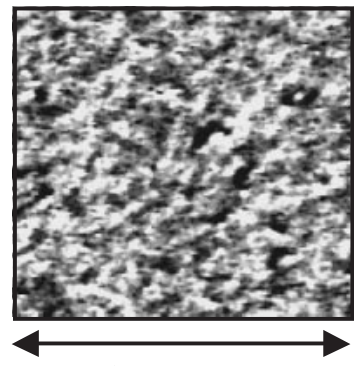

$5.0 \mu \mathrm{m}$ (b) $25^{\circ} \mathrm{C}$

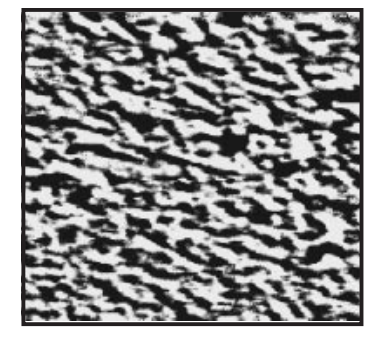

(d) $43^{\circ} \mathrm{C}$

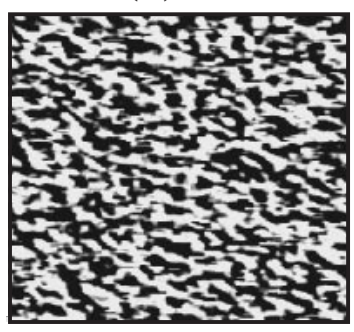

(f) $47^{\circ} \mathrm{C}$

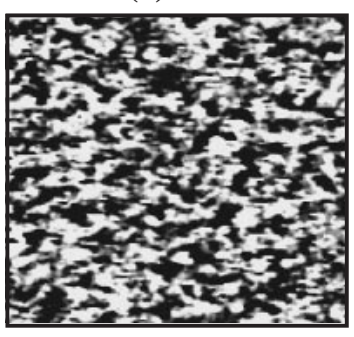

(h) $55^{\circ} \mathrm{C}$

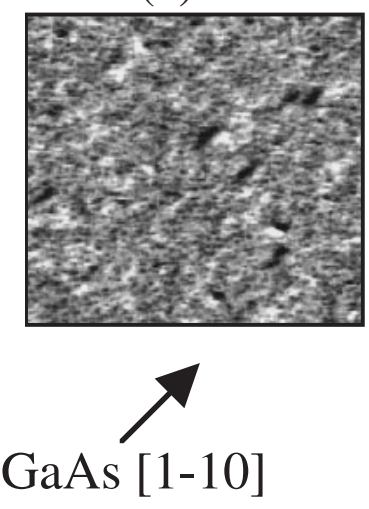

Fig. 3 MFM images of magnetic domain structure in the same area during phase transition of MnAs/GaAs (001) thin film. Magnetization directrion is parallel to [1-10]. Images are taken at each denoted temperature.

to $55^{\circ} \mathrm{C}$ and the same area $\left(5 \times 5 \mu \mathrm{m}^{2}\right)$ of the surface was monitored by AFM/MFM. AFM images did not change during phase transition. It suggests that the spatial changes were too small, viz. atomic level, to detect under such large area observation. Figure 3 shows the change of MFM images from 25 to $55^{\circ} \mathrm{C}$. In MFM images, a domain where the direction of the magnetization is perpendicular to the surface is displayed as a white or black part. ${ }^{13)}$ The white domain has an opposite direction of the magnetization from that of the black domain. Thus, a magnetic domain wall can be formed

around the boundary between the black part and white part. The contrast of MFM images are emphasized to clarify the movement of the magnetic domain structure, only domain walls are viewable in these images. The orientation of magnetization was parallel to GaAs [1-10]. The magnetic domain structures up to $35^{\circ} \mathrm{C}$ did not present large difference at a glance, it became slightly fragmentary. The evident changes come out with the sample temperature of $45^{\circ} \mathrm{C}$. The magnetic domain walls got fuzzy and noises increased. The scratch noises in the Scanning Probe Microscope (SPM) images are generally known as moving objects and/or as an evidence of transformation in progress. The magnetic domain structure lost the directionality at $45^{\circ} \mathrm{C}$ as shown in Fig. 3 . These MFM images prove that the Curie temperature of the present sample is around $45^{\circ} \mathrm{C}$. Above the Curie temperature, the magnetic domains disappeared. The phase transition occurred sharply in a narrow temperature range $\left(<10^{\circ} \mathrm{C}\right)$. The magnetic domain structure changes every second drastically according to the temperature increase between $40^{\circ} \mathrm{C}$ and $50^{\circ} \mathrm{C}$, so that real-time observation of behavior of the magnetic domains during demagnetization is possible if the temperature increase slowly. It is an example of the magnetic structural change controlled by temperature.

\subsection{MnAs on GaAs (111)B}

Figure 4 shows a topographic image (a) and an MFM image (b) of MnAs/GaAs (111)B surface at room temperature. Hexagonal terraces were observed on the surface. Magnetic domain structure does not show directionality and geometric dependence on topography at a glance, but they are still under study. It is found that MnAs/GaAs (111)B is a reasonably good material for nanolithography, because the MnAs film grown on GaAs (111)B is free from rectangle defects and the surface is more flat compared with that on GaAs (001). MFM observation is also difficult after that. It suggests the dependence of magnetic domain structure on topographic structure.

\section{Summary}

We have investigated the magnetic domain structure of epitaxially grown MnAs thin films on GaAs substrate by variable temperature $\mathrm{AFM} / \mathrm{MFM}$ in vacuum. We success-

(a)

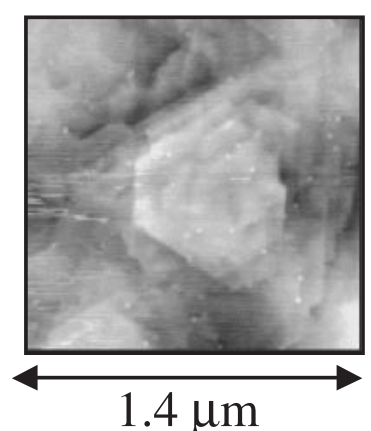

Fig. 4 (a) Topographic image of $1.4 \times 1.4 \mu \mathrm{m}^{2}$ area of $\mathrm{MnAs} / \mathrm{GaAs}$ (111)B thin film. (b) Correspondence MFM image before magnetization. 
fully observed rectangular defects along GaAs [110] direction which disperse randomly on the surface of MnAs/GaAs (001). The visible ferromagnetic-nonmagnetic phase transition occurred in a narrow temperature range between $40^{\circ} \mathrm{C}$ and $50^{\circ} \mathrm{C}$. Local transitions due to the pinning of magnetic poles are observed on the MnAs thin film with rectangular defects. The observation of magnetic domain structure of MnAs/GaAs (111)B is also successfully carried out.

\section{Acknowledgements}

This work was partly supported by NEDO under the Nanotechnology Materials Program.

\section{REFERENCES}

1) S. Miyanishi, H. Akinaga, W. Van Roy and R. Tanaka: Appl. Phys. Lett. 70 (1997) 2046-2048.

2) H. Akinaga, S. Miyanishi, W. Van Roy, J. De Boeck and G. Borghs:
Appl. Phys. Lett. 73 (1998) 3285-3287.

3) M. Mizuguchi, H. Akinaga, K. Ono and M. Oshima: Appl. Phys. Lett. 76 (2000) 1743-1745

4) M. Tanaka, J. P. Harbison, T. Sands, T. L. Cheeks, V. G. Keramidas and G. M. Rothberg: J. Vac. Sci. Technol. B 12 (1994) 1091-1094.

5) M. Tanaka, J. P. Harbison, M. C. Park, Y. S. Park, T. Shin and G. M. Rothberg: Appl. Phys. Lett. 65 (1994) 1964-1966.

6) M. Tanaka, J. P. Harbison, M. C. Park, Y. S. Park, T. Shin and G. M. Rothberg: J. Appl. Phys. 76 (1994) 6278-6280.

7) M. C. Park, Y. Park, T. Shin, G. M. Rothberg, M. Tanaka and J. P. Harbison: J. Appl. Phys. 79 (1996) 4967-4969.

8) C. Kittel: Introduction to Solid State Physics, (Wiley, New York, 1976) p. 439.

9) K. Shigeto, T. Okuno, K. Mibu, T. Shinjo and T. Ono: Appl. Phys. Lett. 80 (2002) 4190-4192.

10) G. Meyer and N. M. Amer: Appl. Phys. Lett. 53 (1988) 1045-1047.

11) M. Yasutake, T. Yamaoka and Y. Nagatani: Jpn. J. Appl. Phys. 35 (1996) 3783-3786.

12) F. Schippan, B. Hehme, L. Daweritz, K. H. Ploog, B. Dennis, K. U. Neumann and K. R. A. Ziebeck: J. Appl. Phys. 88 (2000) 2766-2770.

13) A. Hubert and R. Schäfer: Magnetic Domains, (Springer-Verlag, New York, 1998) p. 78. 